\title{
VALIDITY OF MAXILLARY SINUS VOLUMETRIC MEASUREMENTS USING THE 3D ORTHOGONAL CONE BEAM COMPUTED TOMOGRAPHY IMAGES VERSUS DIRECT SKULL VOLUMETRIC MEASUREMENTS: AN IN VITRO STUDY
}

\author{
Reham Mohamed Hamdy ${ }^{*}$, Reham Ashraf Hussien ${ }^{* *}$ and Moushira Abd-el latif Dahaba ${ }^{* * *}$
}

\begin{abstract}
Introduction: the total volume of maxillary sinus is important for maxillofacial reconstruction, plastic surgeries and prosthetic rehabilitation. It is important to diagnose sinus hypoplasia secondary to craniofacial syndromes, sinus obliteration caused by infections or blood dyscrasis.
\end{abstract}

Aim of the study: The aim of this study was to assess the accuracy of CBCT volumetric measurements of maxillary sinus using the geometric rule of pyramid volume based on CBCT linear measurements versus real skull measurements.

Materials and methods: fourteen skulls were scanned using Planmeca Promax 3D MID and the maxillary sinus pyramid height, length and width were measured in $\mathrm{cm}$. The volume of 24 sinuses were calculated from pyramid volume $\left(\mathrm{cm}^{3}\right)=1 / 3$ Height $\times$ Length $\times$ Width. The CBCT volumes and the reference standard were analyzed for error assessment using Dahelberg error and Relative Dahelberg Error.

Results: 4.3- 4.7\% was the error obtained from CBCT volumetric measurements using the rule of pyramid volume. 0.9 correlation was detected between the $\mathrm{CBCT}$ volume and the reference standard, between the observers and within the same observer.

Conclusion: the geometric rule of pyramid volume based on CBCT linear measurements can be used to assess maxillary sinus volume.

KEY WORDS: Maxillary sinus, CBCT, linear measurements, pyramid volume, accuracy.

* Assistant professor of Oral and Maxillofacial Radiology, Faculty of Dentistry, Cairo University.

** Assistant lecturer of Oral and Maxillofacial Radiology, Faculty of Dentistry, Cairo University.

*** Professor of Oral and Maxillofacial Radiology, Faculty of Dentistry, Cairo University. 


\section{INTRODUCTION}

Since the introduction of CBCT to the field of 3D imaging, many applications have become possible in dentistry ${ }^{(1)}$. The interactive CBCT software and its different functional tools made volumetric measurements more vivid ${ }^{(2,3)}$. Manual and semiautomatic segmentation of maxillary sinus, which depends on linear tracing of the sinus boundaries, is time consuming and operator dependent ${ }^{(2)}$. Automatic segmentation of maxillary sinus, which depends on air thresholding, excludes mucosal thickening of the Schneiderian membrane causing faulty measurements of the sinus volume ${ }^{(3)}$.

The geometric method to calculate the volume of maxillary sinus has been always applicable and depends on the fact that the sinus is a square base pyramid $^{(4,5)}$. The volume of a square based pyramid is calculated from the geometric rule $1 / 3$ pyramid height $\times$ pyramid base Length $\times$ pyramid base width $^{(5)}$. To the best of the authors' knowledge the validity/ accuracy of the geometric method to calculate the volume of maxillary sinus didn't receive much attention ${ }^{(5,6)}$. For so, this study was aimed to assess the validity of the geometric method to calculate the volume of maxillary sinus using CBCT.

\section{MATERIALS AND METHODS}

This study was performed on 14 dry human skulls obtained from the Anatomy department, Faculty of Medicine, Cairo University. The skulls identity regarding age, gender and race was of no value in this study. The skulls enrolled in the study fulfilled a major inclusion criterion that was intact anterior, posterior and lateral walls as well as intact roof and floor of maxillary sinus. Both dentulous and edentulous skulls were included. Skulls with gross surface defect, traumatic injuries, apparent diseases or space occupying lesions and severe mutilation caused by environmental decay were excluded if they affected the maxilla and the maxillary sinus.

\section{Skull preparation}

Simulation of soft tissue attenuation characteristic was achieved by applying 20 layers of pink modeling wax (Cavex, Holand BV modeling wax) to reach $14 \mathrm{~mm}$ thickness on the outer surface of the skull while the mandible and maxilla were fixed in centric position ${ }^{(7)}$.

\section{CBCT scanning}

The skulls were stabilized in the scan position using tripod, the chin and the lateral head support of the CBCT machine (Planmeca Promax 3D MID, Hilinski, Finland). The skulls were orientated with its midsagittal plane coinciding with the CBCT machine vertical laser beams and the occlusal plane was parallel to the horizontal plane. The skulls were scanned using $20 \times 17 \mathrm{~cm}$ FOV, $400 \mu \mathrm{m}$ voxel resolution and $90 \mathrm{KVp}, 8 \mathrm{~mA}$, exposure time $13.5 \mathrm{sec}$.

\section{CBCT linear measurements and volumetric cal- culation of maxillary sinus (test method)}

The CBCT data set was exported from the workstation to a personal computer using the DICOM file format (Digital Imaging and Communication in Medicine). The CBCT machine's software, Planmeca Romexis viewer 3.8.1.R software was used in linear measurements. The linear measurements were conducted on the basic orthogonal CBCT images: axial, coronal and sagittal images using the built-in ruler of the software. As the maxillary sinus assumes 3D pyramid shape, three dimensions of the pyramid were measured ${ }^{(5)}$. The three dimensions representing the pyramid of the maxillary sinus were: (1) pyramid base length, craniocaudal dimension (CC) of the medial wall of the sinus, which extends from the highest point of the maxillary sinus anterior to the top of the pterygomaxillary fissure to the most inferior point of the maxillary sinus floor located in the alveolar process (figure 1A), (2) pyramid base width, antro-posterior dimension (AP) of the medial wall of the sinus which extends from the most anterior point to the most posterior point of the sinus (figure 1B), (3) pyramid height, medio-lateral dimension (ML) of the maxillary sinus, which extends from the most lateral point of the pyramid apex located in the zygomatic bone 
perpendicular to the pyramid base representing the medial wall of the sinus (figure 1C). The sinus volume was calculated from the geometric equation of square base pyramid volume $\left(\mathrm{cm}^{3}\right)=1 / 3$ Height $\times$ Length $\times$ Width $(\mathrm{cm})^{(5)}$.

By scrolling the sagittal images, the most lateral point of maxillary sinus apex and the most medial point of maxillary sinus at its medial wall were identified and a perpendicular line was dragged between the same two points on the coronal and axial images. By scrolling the coronal images, the most posterior point of maxillary sinus at its posterior wall and the most anterior point of maxillary sinus at the junction between the medial and anterior walls were identified and a perpendicular line was dragged between the same two points on the sagittal image. By scrolling the axial images, the most superior point of maxillary sinus at its roof and the most inferior point of maxillary sinus at the alveolar process were identified and a perpendicular line was dragged between the same two points on the sagittal image (figure $1 \mathrm{~A}, \mathrm{~B}, \mathrm{C}$ ).

Volumetric measurement of maxillary sinus impression using water displacement technique (reference standard)

The reference standard in this study was the indirect volume measurements of the maxillary sinus rubber impression using fluid displacement technique of the recorded rubber impression following Arquimedes principle ${ }^{(8)}$.

To fill the maxillary sinus with rubber impression, an access opening was drilled in its anterior wall $2 \times 2 \mathrm{~cm}$ from the canine fossa. Before drilling, high viscosity condensation silicon (Zetaplus Zhermack, UK) was used to take a rubber impression of the anterior wall of the maxillary sinus to keep a record of its anatomy for later use. The used high viscosity condensation silicon had 2 minutes working time, 6 minutes setting time and $-0.10 \%$ polymerization shrinkage. After drilling the access opening, the maxillary sinus was filled with polyether hydrophilic impression material, soft medium bod- ied consistency, regular setting (3M ESPE monophase Impregum, USA) which had 2.45 minutes working time, 6 minutes setting time and $-0.001 \%$ polymerization shrinkage. $3 \mathrm{M}$ ESPE Pentamix ${ }^{\mathrm{TM}} 3$ mixing dispenser was used to mix and to deposit the Impregum within the sinus. To guarantee proper filling of the sinus with the rubber impression material, gentle tapping over the skull was applied. The impression of the anterior wall of the maxillary sinus- taken earlier before drilling- was replaced over the access opening after complete filling of the sinus to restore the continuity of the drilled area.

After complete setting of the rubber impression, the skull was scanned using CBCT once more using the same parameters. The CBCT images were scrolled and the extension of the rubber impression within the maxillary sinuses was inspected to detect areas of under filling or over extension. Bone segments surrounding areas of under filling were preserved during breaking the skull for further rubber addition and areas of over extension were trimmed.

The rubber impression of each maxillary sinus was immersed in a $200 \mathrm{ml}$ measuring beaker filled to a $100 \mathrm{ml}$ mark with tape water at room temperature. Then, the volume of the displaced water -which equals the volume of the maxillary sinus impressionwas withdrawn using $5 \mathrm{ml}$ measuring pipette with an accuracy of $0.01 \mathrm{ml}$ till the initial level of water before impression immersion was reached and transferred into $100 \mathrm{ml}$ measuring cylinder with an accuracy of $10 \mathrm{ml}$ and the volume of the displaced water was quantified.

The CBCT measurements were conducted by two radiologists. The first radiologist conducted the CBCT measurements twice separated by two weeks interval. The second radiologist conducted the CBCT measurements only once. The first and the second radiologists were blinded from the readings of the other radiologist and were blinded from the reference standard measurements during CBCT measurements. The third radiologist performed the measurements of the reference standard. 


\section{Statistical analysis}

Using $\mathrm{G}^{*}$ Power version 3.1.9.2, a power analysis was designed to achieve adequate power to apply a two-sided statistical test of the research hypothesis (null hypothesis) that there was no difference in the accuracy of CBCT volumetric measurements and the reference standard maxillary sinus volume. According to the results of Alves Jr, Matheus, et al. (9) assuming an alpha $(\alpha)$ level of $0.05(5 \%)$, a Beta ( $\beta$ ) level of $0.20(20 \%)$ i.e. power $=80 \%$, an effect size (d) of (1.43) and a (Mean \pm Standard deviation) of (20689.48 \pm 123.86$)(20514.16 \pm 1.75)$ for both measurements; The predicted sample size (n) was found to be a total of (21) sinuses.

All Data were collected, tabulated and subjected to statistical analysis. Statistical analysis was performed by SPSS in general (version 17), also Microsoft office Excel was used for data handling and graphical presentation. Quantitative variables were described by the Mean, Standard Deviation (SD). Qualitative categorical variables were described by Percentages. For assessment of the agreement of volumetric measurements of maxillary sinus obtained by CBCT scanner and both inter and intra observer reliability analysis, Dahelberg error (DE) and Relative Dahelberg Error (RDE) were used together with Concordance Correlation Coefficients (CCC) including the $95 \%$ confidence limits of the coefficient. To measure and quantify the size of the differences, Bland and Altman 95\% confidence Limits of Agreements (LOA) were applied.

\section{RESULTS}

The relative Dahlberg error showed small error 4.3- $4.7 \%$ between the mean volume of the maxillary sinus calculated from the $\mathrm{CBCT}$ linear measurements using the geometric rule of pyramid volume and the mean of the real volume of the maxillary sinuses using the water displacement technique. The concordance correlation coefficient showed excellent correlation and equivalence 0.98 between the test method and the reference standard. The mean difference between the test method and the reference standard indicated a tendency of the test method to underestimate the volume of the maxillary sinus. The $95 \%$ confidence interval was limited between $-3.08-3.59$ (table 1).

The maximum relative error between the observers was detected in the medio-lateral dimension $4.6 \%$ followed by the antro-posterior dimension $4.5 \%$. Excellent intra-observer agreement was detected in the three dimensions measured: 0.98 for antro-posterior dimension, 0.97 for craniocaudal dimension, 0.99 for medio-lateral dimension. Interobserver agreement was $0.87,0.91$ and 0.94 for the same dimensions respectively (table 2).

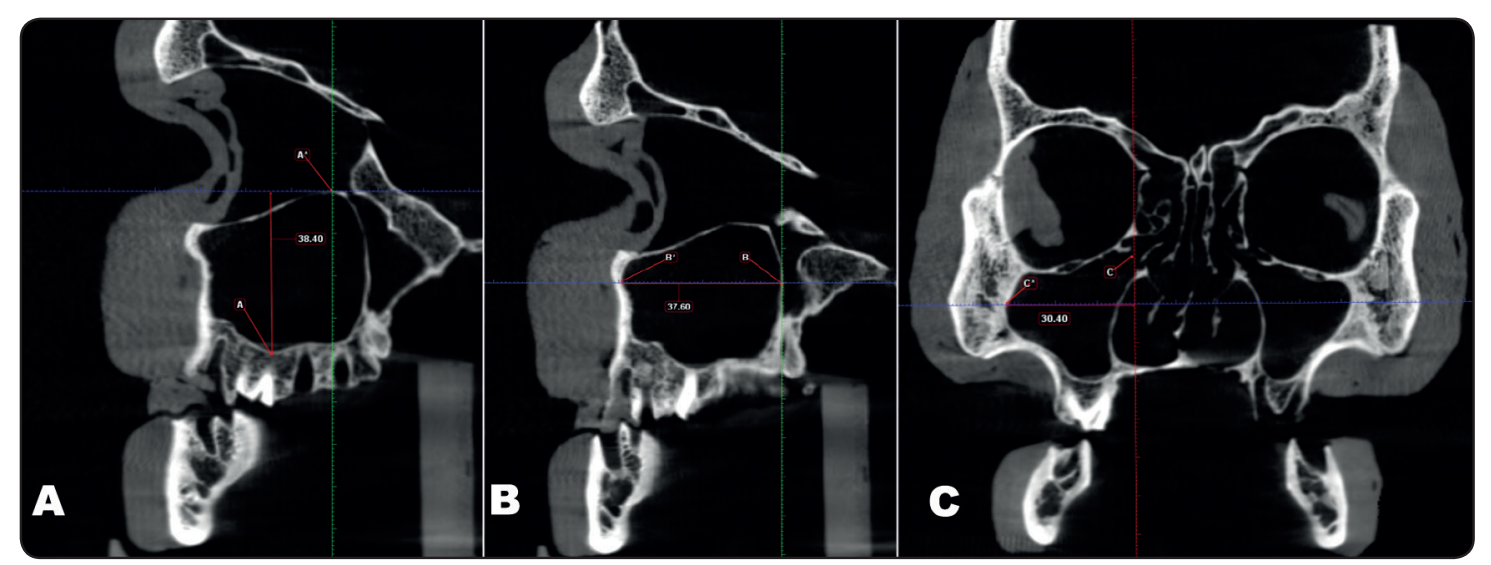

Fig. (1) (A, B) sagittal CBCT images showing point A is the most inferior point of the maxillary sinus, point $\mathrm{A}^{*}$ is the most superior point of the maxillary sinus and the linear measurements between $\mathrm{A}, \mathrm{A}^{*}$ is the $\mathrm{CC}$ dimension of the maxillary sinus, point $\mathrm{B}$ is the most posterior point of the maxillary sinus, point $\mathrm{B} *$ is the most anterior point of the sinus, and the linear measurements between $\mathrm{B}, \mathrm{B} *$ is $\mathrm{AP}$ dimension of the maxillary sinus. $(\mathrm{C})$ coronal $\mathrm{CBCT}$ image showing point $\mathrm{C}$ is the most medial point of the sinus, point $\mathrm{C}^{*}$ is the most lateral point of the sinus, and the linear measurement between $\mathrm{C}, \mathrm{C}^{*}$ is the ML dimension of the maxillary sinus. 
TABLE (1) Error assessment of maxillary sinus volume measurements between CBCT (test method) and the reference standard

\begin{tabular}{|c|c|c|c|c|c|c|c|c|c|c|c|}
\hline & & & & & & & \multirow{2}{*}{\multicolumn{2}{|c|}{$\begin{array}{c}\text { Bland \& Altman } \\
\text { Limits of } \\
\text { Agreement (LOA) } \\
\begin{array}{c}95 \% \text { confidence } \\
\text { limits }\end{array}\end{array}$}} & \multicolumn{3}{|c|}{$\begin{array}{c}\text { Concordance Correlation } \\
\text { Coefficient }\end{array}$} \\
\hline & & & & & & & & & \multicolumn{3}{|c|}{$95 \%$ confidence limits } \\
\hline $\begin{array}{c}\text { Volume } \\
\text { measurements }\end{array}$ & $\begin{array}{l}\text { Mean } \\
\mathrm{Cm}^{3}\end{array}$ & SD & $\begin{array}{c}\text { Dahlberg } \\
\text { error/ DE } \\
\mathrm{Cm}^{3}\end{array}$ & $\begin{array}{c}\text { Relative } \\
\text { Dahlberg } \\
\text { Error/ } \\
\text { RDE\% }\end{array}$ & $\begin{array}{l}\text { Mean of } \\
\text { Difference } \\
\text { (Reference } \\
\text { - Test) } \mathrm{Cm}^{3}\end{array}$ & $\begin{array}{l}\text { SD of the } \\
\text { Difference }\end{array}$ & Lower & Upper & $\mathrm{CCC}$ & Lower & Upper \\
\hline Reference & 12.86 & 5.08 & \multirow{2}{*}{0.60} & \multirow{2}{*}{$4.7 \%$} & \multirow{2}{*}{0.26} & \multirow{2}{*}{1.70} & \multirow{2}{*}{-3.08} & \multirow{2}{*}{3.59} & \multirow{2}{*}{0.985} & \multirow{2}{*}{0.972} & \multirow{2}{*}{0.992} \\
\hline V1-O1R1 & 12.60 & 4.88 & & & & & & & & & \\
\hline Reference & 12.86 & 5.08 & \multirow{2}{*}{0.59} & \multirow{2}{*}{$4.6 \%$} & \multirow{2}{*}{0.23} & \multirow{2}{*}{1.71} & \multirow{2}{*}{-3.12} & \multirow{2}{*}{3.57} & \multirow{2}{*}{0.986} & \multirow{2}{*}{0.973} & \multirow{2}{*}{0.992} \\
\hline V2-O1R2 & 12.63 & 4.91 & & & & & & & & & \\
\hline Reference & 12.86 & 5.08 & \multirow{2}{*}{0.56} & \multirow{2}{*}{$4.3 \%$} & \multirow{2}{*}{0.17} & \multirow{2}{*}{0.72} & \multirow{2}{*}{-1.24} & \multirow{2}{*}{1.58} & \multirow{2}{*}{0.987} & \multirow{2}{*}{0.976} & \multirow{2}{*}{0.992} \\
\hline $\mathrm{V} 3-\mathrm{O} 2$ & 12.69 & 4.72 & & & & & & & & & \\
\hline
\end{tabular}

TABLE (2) Intra-observer and inter-observer reliability

\begin{tabular}{|c|c|c|c|c|c|c|c|c|c|c|c|}
\hline & & & & & & & \multirow{2}{*}{\multicolumn{2}{|c|}{$\begin{array}{c}\begin{array}{c}\text { Bland \& Altman } \\
\text { Limits of Agreement } \\
\text { (LOA) }\end{array} \\
\begin{array}{c}95 \% \text { confidence } \\
\text { limits }\end{array}\end{array}$}} & \multicolumn{3}{|c|}{$\begin{array}{c}\text { Concordance Correlation } \\
\text { Coefficient }\end{array}$} \\
\hline & & & & & & & & & \multicolumn{3}{|c|}{$95 \%$ confidence limits } \\
\hline $\begin{array}{c}\text { Linear } \\
\text { measurements }\end{array}$ & $\begin{array}{c}\text { Mean } \\
\mathrm{Cm}\end{array}$ & SD & $\begin{array}{c}\text { Dahlberg } \\
\text { error/ DE } \\
\quad \mathrm{Cm}\end{array}$ & $\begin{array}{c}\text { Relative } \\
\text { Dahlberg } \\
\text { Error/ } \\
\text { RDE } \\
\% \\
\end{array}$ & $\begin{array}{c}\text { Mean } \\
\text { Difference of } \\
\text { the observer's } \\
\text { readings } \mathrm{Cm}\end{array}$ & $\begin{array}{l}\text { SD of the } \\
\text { Difference }\end{array}$ & Lower & Upper & $\mathrm{CCC}$ & Lowe & Upper \\
\hline AP1-O1 & 36.53 & 5.02 & \multirow{2}{*}{0.53} & \multirow{2}{*}{$1.5 \%$} & \multirow{2}{*}{0.00} & \multirow{2}{*}{1.59} & \multirow{2}{*}{-3.11} & \multirow{2}{*}{3.11} & \multirow{2}{*}{0.988} & \multirow{2}{*}{0.977} & \multirow{2}{*}{0.993} \\
\hline AP2-O1 & 36.53 & 4.82 & & & & & & & & & \\
\hline AP1-O1 & 36.53 & 5.02 & \multirow{2}{*}{1.65} & \multirow{2}{*}{$4.5 \%$} & \multirow{2}{*}{-0.39} & \multirow{2}{*}{10.84} & \multirow{2}{*}{-21.64} & \multirow{2}{*}{20.85} & \multirow{2}{*}{0.871} & \multirow{2}{*}{0.774} & \multirow{2}{*}{0.927} \\
\hline AP3-O2 & 36.93 & 4.27 & & & & & & & & & \\
\hline $\mathrm{CC} 1-\mathrm{O} 1$ & 35.93 & 4.42 & \multirow{2}{*}{0.72} & \multirow{2}{*}{$2.0 \%$} & \multirow{2}{*}{-0.24} & \multirow{2}{*}{4.39} & \multirow{2}{*}{-8.85} & \multirow{2}{*}{8.37} & \multirow{2}{*}{0.974} & \multirow{2}{*}{0.953} & \multirow{2}{*}{0.986} \\
\hline $\mathrm{CC} 2-\mathrm{O} 1$ & 36.17 & 4.74 & & & & & & & & & \\
\hline $\mathrm{CC} 1-\mathrm{O} 1$ & 35.93 & 4.42 & \multirow{2}{*}{1.39} & \multirow{2}{*}{$3.9 \%$} & \multirow{2}{*}{0.08} & \multirow{2}{*}{6.00} & \multirow{2}{*}{-11.69} & \multirow{2}{*}{11.84} & 0012 & 0846 & 0051 \\
\hline $\mathrm{CC} 3-\mathrm{O} 2$ & 35.85 & 5.12 & & & & & & & 0.915 & 0.040 & 195. \\
\hline ML1-O1 & 27.43 & 5.42 & (20 & $10 \%$ & 005 & $0-41$ & 074 & 085 & 0007 & 0004 & 0008 \\
\hline ML2-O1 & 27.37 & 5.35 & 0.23 & 1.110 & $0.0 \mathrm{~J}$ & 0.41 & -0.14 & 0.03 & 0.991 & 0.994 & 0.990 \\
\hline ML1-O1 & 27.43 & 5.42 & & & & & & & & & \\
\hline ML3-O2 & 27.34 & 5.26 & 1.20 & 4.070 & 0.0 & 5.4 & -0.04 & 0.02 & 0.942 & 0.03 & 0.90 \\
\hline
\end{tabular}




\section{DISCUSSION}

The study in hand was conducted to assess the validity of CBCT volumetric measurements of the maxillary sinus in comparison with the real measurements from dry human skulls. The study was carried on 28 maxillary sinuses of 14 dry human skulls. CBCT volumetric measurements of the maxillary sinus were calculated using the geometric rule to calculate the volume of square base pyramid, while the real volumetric measurements were performed indirectly using the water displacement technique.

The results of this study showed excellent to good inter-observer agreement of $0.9-0.8$ in the three dimensions of the maxillary sinus measured from CBCT to calculate the volume of the maxillary sinus which denoted reproducible methodology implemented in measuring the dimensions of the maxillary sinus and hence calculating its volume. Excellent intra-observer agreement 0.9 revealed repeatability of the three measurements. Both the inter and intra-observer agreement ascertained the simple, easy, standardized and reproducible methodology of measuring the maxillary sinus dimensions and hence its volume. Scrolling the maxillary sinus in the three dimensions antroposterior, craniocaudal and medio-lateral was easy and applicable way to identify the furthest anatomical points intended for each of the three linear measurements required to calculate the sinus volume.

Moreover, the greatest absolute error and the relative percentage error between the two observers was detected in the maxillary sinus height which is the ML dimension of the sinus and was $1.26 \mathrm{~cm}^{3}$ and $4.6 \%$. This error was less than the $5 \%$ clinically accepted error ${ }^{(10)}$. To explain the reason behind such greatest error in the sinus height, the two observers found difficulty to identify the most medial point of the sinus which was located in the middle conchae in the majority of cases. Anatomically, the nasal conchae are very thin, wispy and hollow so they easily brake dawn and separate from dry human skulls secondary to environmental decay. Gross examination of the middle conchae, which are hidden and invisible to be examined clinically, was not possible and made exclusion of those skulls difficult.

The results of this study further showed excellent agreement 0.98 between the mean volume of maxillary sinus calculated using the geometric rule of square base pyramid based on CBCT linear measurements and the real volumetric measurements. Furthermore, the absolute error and the relative percentage error of CBCT volumetric measurements was $0.56-0.6 \mathrm{~cm}^{3}$ and $4.3-4.7 \%$ respectively and were less than the $5 \%$ clinically accepted error ${ }^{(10)}$. Moreover, the mean difference between the geometric method and the reference standard showed tendency of the test method to under estimate the volume of the sinus by $0.17-0.26 \mathrm{~cm}^{3}$. This could be referred to the difference between the regular nature of 3D shapes as the pyramid and the real nature of the maxillary sinus. The maxillary sinus closely resembles a pyramid but it doesn't exactly fit. The maxillary sinus has rounded corners and angles and croaked surfaces unlike the regular 3D shape of the pyramid which has sharp angles, lines, vertices and flat surfaces. A systematic error with a confidence interval between -1.24 and 3.59 could be expected if other studies would be conducted following our methodology to calculate the maxillary sinus volume.

In agreement, Agnieszka et al ${ }^{(5)}$ compared the volume of maxillary sinus calculated from mathematical formulae of sphere, pyramid, and the mean of both sphere and pyramid and compared them with automatically segmented sinus from multi slice CT scans obtained using 128-slice CT scanner; Somatom Definition AS+ (Siemens Healthcare) and Syngo Via for Oncology Siemens software. The CT scans were obtained respectively for patients 
suspected to have traumatic injuries or neurological diseases. Their results showed 0.99 correlation coefficient and no statistically significant difference was found between the automatically segmented volume of maxillary sinus and the mathematically calculated volume using the sphere, pyramid formulae and the mean. They didn't report the absolute or relative error in their results. However, they reported that the pyramid formula gave smaller volume, the sphere formula gave bigger volume and the mean of both was closest to the automatically segmented volume. For which the concluded that the maxillary sinus shape lies between a sphere and a pyramid.

The study by Sahlstrand Johnson et al $2011^{(6)}$ calculated the volume of the maxillary sinuses from the equation width $\times$ anteroposterior $\times$ craniocaudal diameter of the sinus $\times 0.5$ and automatically estimated sinus volume using Leonardo work station volume application for segmentation both volumes were conducted on scans obtained by multi slice Siemens CT scanner. Similar to our results, their results showed almost perfect agreement 0.90-0.93, good concordance correlation and a random error of 1.9-2.4 $\mathrm{cm}^{3}$ between the calculated and automatically estimated volume of the sinus. However, their results showed $14-17 \%$ random error between the automatically segmented volumes and the calculated volumes. Mor eover, they reported that calculated volume of the sinus based on the three diameters gave an app roximate value that could be beneficial in clin ical practice when CT software were not available and that the calculated volume was not suitable for research study. The authors of this study beli eved that the quotient used to calculate the sinu s volume from the three diameters in Sahlstrand Johnson et al study ${ }^{(6)}$ was not justified or explained in their discussion and had no geometric reference compared to the equation of sphere and pyramid used in Agnieszka et al ${ }^{(5)}$ work and our work. Moreover, the anterior measurement of the sinus in Sahlstrand Johnson et al study ${ }^{(6)}$, which was an average of two measurements taken at the anterior wall of the sinus and the middle of the sinus, might be the reason behind the 14-17\% random error of the calculated volume. In addition, the use of paired sample t-test to calculate random error of the difference was questionable. ${ }^{(11)}$

The bright aspect of this study was to calculate the volume of maxillary sinus using a simple geometric equation of regular 3D shape volume. This method was time saving, e asy to be applied and reproducible. Moreover, this study used maxillary sinus rubber impression as a reference standard not manual or automatic segmentation to validate the test method. Furthermore, the use of correlation coefficient to assess the agreement between the test and reference standard and the limits of agreement to quantify the systematic error were the correct statistical test for data analysis. The use of standardized method to calculate the sinus volume had been high lightened by Giacomini et $\mathrm{al}^{(11)}$. The use of reference standard and proper statistical analysis was milestone in accuracy studies that was addressed in the systematic review conducted by Alsufyani et al (12) and for which he couldn't reach consensus regarding volumetric measu rement of upper air way using CBCT. Yet, the geometric method to calculate the maxillary sinus volume can't provide any data about the surface morphology or sinus topography as the CBCT segmentation software can.

\section{CONCLUSION}

All the aforementioned results proved that the geometric rule of square base pyramid was valid to calculate the maxillary sinus volume from CBCT 3D orthogonal images and that the methodology implemented in the measurements was reproducible.

\section{RECOMENDATION}

Similar accuracy studies should be conducted to calculate the maxillary sinus volume from CBCT basic orthogonal images using sphere volume. 


\section{REFERENCES}

1. Carter L, Farman AG, Geist J, et al. American Academy of Oral and Maxillofacial Radiology Executive Opinion Statement on Performing and Interpreting Diagnostic Cone Beam Computed Tomography. Oral Surg Oral Med Oral Pathol Oral Radiol Endod. 2008; 106: 561-62. DOI: $10.1016 /$ j.tripleo.2008.07.007

2. Neelapu BC, Kharbanda OP, Sardana HK, Gupta A, Vasamsetti S, Balachandran R, Rana SS, Sardana V. The reliability of different methods of manual volumetric segmentation of pharyngeal and sinonasal subregions. Oral surgery, oral medicine, oral pathology and oral radiology 2017; 124 (6): 577-587. DOI10.1016/j.oooo.2017.08.020

3. Shi, H., Scarfe, W.C. \& Farman, A.G. Maxillary Sinus 3D Segmentation and Reconstruction from Cone Beam CT Data Sets. Int J CARS 2006; 1: 83-89. https://doi. org/10.1007/s11548-006-0041-9

4. Souza AD, Rajagopal KV, Ankolekar VH, Souza AD, Kotian SR. Anatomy of maxillary sinus and It's ostium: A radiological study using computed tomography. CHRISMED Journal Health Research 2016; 3: 37-40. DOI: $10.4103 / 2348-3334.172397$

5. Agnieszka P, Tomasz K, Artur R, Alicja S, Katarzyna JP, Dorota LM, Krzysztof G, Agata CJ. Introducing a simple method of maxillary sinus volume assessment based on linear dimensions. Annals of Anatomy 2018; 215: 47-51. https://doi.org/10.1016/j.aanat.2017.09.010

6. Sahlstrand-Johnson P, Jannert M, Strömbeck A, Abul-Kasim
K. Computed tomography measurements of different dimensions of maxillary and frontal sinuses. BMC Med Imaging 2011; 11:8. Doi:https://doi.org/10.1186/1471-2342-11-8

7. Schropp L, Alyass NS, Wenzel A, Stavropoulos A. Validity of wax and acrylic as soft tissue simulation materials used in in-vitro radiographic studies. Dentomaxillofacial Radiology. 2012; 000:1-5

8. Coronado C, Arriagada O, Galdames IS. Easy and Unbiased Determination of the Maxillary Sinus Volume. International Journal of Morphology 2011; 29(4):1375-1378.

9. Alves Jr, Matheus, et al. Is the airway volume being correctly analyzed? American Journal of Orthodontics and Dentofacial Orthopedics 2012; 141: 657-661.

10. Tarazona Alvarez P, Romero Millan J, Penarrocha Oltra D, Fuster Torres M, Tarazona Alvarez B, Penarrocha Diago M. Comaprative study of mandibular linear meaasurements obtained by cone beam computed tomography and digital calipers. Journal of Clin Exp Dent. 2014; 6 (3): 4-7.

11. Giacomini G, Pavan ALM, Altemani JMC, Duarte SB, Fortaleza $\mathrm{CMCB}$, et al. Computed tomography-based volumetric tool for standardized measurement of the maxillary sinus. PLOS ONE 2018; 13(1): e0190770. https://doi. org/10.1371/journal.pone.0190770

12. Alsufyani NA, Flores-Mir C, Major PW. Three-dimensional segmentation of the upper airway using cone beam CT: a systematic review. Dento maxillo facial radiology. 2012;41(4):276-84. pmid:22517995; PubMed Central PMCID: PMC3729002. 